\title{
Viewpoint
}

\section{A rheumatological dilemma: is it possible to modify the course of rheumatoid arthritis? Can we answer the question?}

T PULLAR AND H A CAPELL
From the University Department of Medicine, Centre for Rheumatic Diseases, Wards 14/15 Royal Infirmary,
Glasgow G4 OSF

SUMmaRY The question 'Does the use of second-line therapy confer long-term benefit on음 outcome measures in rheumatoid arthritis?' remains unanswered. The major obstacle whichprevents collection of the necessary data is the lack of a suitable control group. In this reporto experience with three 'second-line placebo groups' is described, and previous studies in the literature which incorporated a placebo group are reviewed. In the absence of concurrent婞 corticosteroid therapy very few patients remain on placebo second-line medication after one $\overrightarrow{0}$ year. Those that do, appear to have milder disease and are not representative of the group asoago whole. Data on outcome measures need to be collected over two to five years, but the answer the question which is posed does not depend upon larger and larger placebo groups whitho constitute increasing bias. To define the extent of benefit offered by the more powerful therapeutic agents a novel approach in regard to drug assessment will be required.

Key words: second-line drugs, placebo.

Gold salts have been used in the treatment of rheumatoid arthritis (RA) for almost 60 years, but rheumatologists are still uncertain about the longterm effect on the course of the disease. This is equally true of other 'second-line' drugs, such as penicillamine, sulphasalazine, or the cytotoxic agents.

Both early placebo controlled trials ${ }^{12}$ of these drugs and subsequent studies provided many shortterm answers. Why, then, has long-term evaluation been so unsatisfactory? Several reasons have been suggested, including poor trial design, inadequate patient numbers in individual studies, disproportionate concern with 'process' rather than 'outcome' measurements, and medical unwillingness to enrol rheumatoid patients into a study which would contain a placebo group. To some extent such factors have been contributory. However, the very nature of rheumatoid disease and the magnitude of

Accepted for publication 15 November 1984.

Correspondence to Dr T Pullar. symptomatic response to therapy have posed sub? stantial problems in assessment of disease activityo and in the maintenance of a placebo group.

In an attempt to generate placebo data over prolonged periods three recent short-term second line studies ${ }^{3-6}$ have been continued beyond theo initial six-month assessment period.

\section{Patients and methods}

Entry criteria to all three studies were identical $: \stackrel{N}{\circ}$ namely patients with active definite or classicah rheumatoid arthritis, ${ }^{7}$ which was not adequately controlled by non-steroidal anti-inflammatory drugs (NSAID). No patient had received corticosteroids or second-line drugs in the three months before entry, and none had previously received any of thes agents under evaluation. Patients were encouraged to continue the same dose of NSAID throughout the study, and no patients received systemic corticoster- $-\vec{\Phi}$ oids. Clinical assessments were carried out by के 
metrologist (research nurse) who was 'blind' to the patient's therapy.

STUDY 1

Ninety patients were randomly allocated to auranofin $3 \mathrm{mg}$ twice daily, matching placebo, or intramuscular sodium aurothiomalate (GST) and assessed at 12,24 , and 48 weeks.

STUDY 2

Sixty patients were randomly allocated to either ketotifen $1 \mathrm{mg}$ twice daily or matching placebo and assessed at 12 and 24 weeks.

\section{STUDY 3}

Ninety patients were randomly allocated to either sulphasalazine (salicylazosulphapyridine (SASP)) 3 g/day, matching placebo, or intramuscular GST and assessed at 12, 24, and 48 weeks.

Patients in the GST groups did not receive placebo tablets, nor did those patients allocated to oral treatment receive placebo injections. In study 1 the physician (but not the metrologist) was aware of the patient's treatment allocation, and in study 3 the physician was aware whether the patient received tablets or injections but did not know the nature of the tablets. Although analysis was carried out at 24 weeks to assess efficacy, only the computer operator was aware of the 'coding', and blindness was not affected.

Statistical analysis was carried out using appropriate non-parametric statistics.

\section{Results}

Within each study patients were comparable with respect to age, disease duration, and disease severity (Kruskal Wallis $\mathrm{p}>0 \cdot 05$ ). Although there were no formal entry criteria to these studies other than physician and patient agreement that rheumatoid arthritis was active and uncontrolled on NSAID therapy, retrospective analysis of clinical and laboratory data indicates that all patients had moderate or severe disease, and none had mild disease. $^{8}$

Table 1 shows the number of patients remaining on each form of therapy at 24 and 48 weeks, and the reasons for discontinuation of therapy are shown in Table 2. In the placebo and ketotifen groups (the latter drug proved not to have a second-line effect) inefficacy was the most common reason for cessation of therapy. Adverse reaction accounted for the majority of drop outs on the active drugs and were of the type expected for these drugs. All patients recovered from their side effects.

The only favourable change which occurred in the placebo group was in study 2 where both the ketotifen and placebo groups showed significant improvement in articular index. This subjective change was associated with a change in metrologist. All other placebo parameters showed no benefit despite the fact that patients who were presumably deteriorating more rapidly were able to drop out because of lack of effect.

Table 3 shows within-group comparisons and Table 4 a between-group comparison of inflammatory indices at 24 and 48 weeks. The number of patients assessed in Tables 3 and 4 is not necessarily the same as the number shown in Table 1 , as results were analysed for patients who dropped out on the

Table 1 Number of patients remaining on treatment for 24 and 48 weeks

\begin{tabular}{|c|c|c|c|c|c|c|c|c|}
\hline & \multicolumn{3}{|l|}{ Study $I$} & \multicolumn{2}{|l|}{ Study 2} & \multicolumn{3}{|c|}{ Study 3} \\
\hline & Auranofin & $G S T$ & Placebo & Ketotifen & Placebo & $S A S P$ & $G S T$ & Placebo \\
\hline No entered & 30 & 30 & 30 & 30 & 30 & 30 & 30 & 30 \\
\hline No on treatment at 24 weeks & 26 & 23 & 17 & 19 & 11 & 18 & 18 & 14 \\
\hline No on treatment at 48 weeks & 17 & 22 & 0 & 2 & 1 & 12 & 12 & 6 \\
\hline
\end{tabular}

Table 2 Reasons for discontinuing therapy

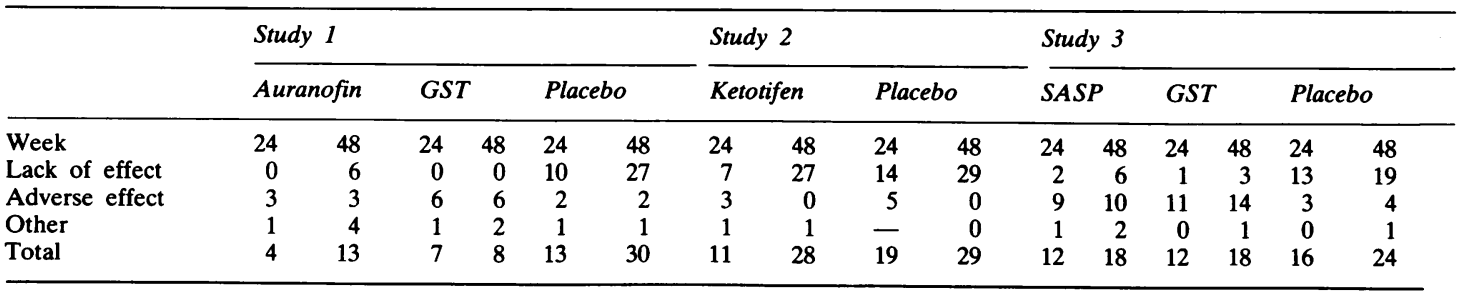


24-week visit but who underwent 24-week assessment.

In study 3 initial erythrocyte sedimentation rate (ESR) in patients who continued placebo for 48 weeks differed significantly from those who discontinued placebo (Mann-Whitney $U$ test $p=0.05$ ) and was also significantly lower than in those who continued sulphasalazine $(\mathrm{p}<0.05)$ but not GST $(p>0 \cdot 05)$.

REVIEW OF PREVIOUS PLACEBO STUDIES The earliest placebo controlled trials of second-line therapy were concerned with courses of gold treatment $^{12}$ rather than the long-term treatment regimen now employed. These studies therefore are not relevant to the present problem.

Two placebo controlled trials of intramuscular (IM) gold salts have been carried out using modern treatment regimens. In the study carried out by the cooperating clinics (seven centres) 936 patients were treated with IM gold salts, and 32 received placebo injections. Twenty-two patients receiving gold and 21 patients receiving placebo remained on treatment at six months, while one gold treated and eight placebo treated patients had stopped over this period because of lack of effect. A significant improvement was seen in ESR (but not other parameters) in the gold group. After six months those patients remaining on gold were randomly므. allotted to gold or placebo, and assessment after a further 12 months showed a trend in favour of gold. Systematic corticosteroids were not allowed during this study.

The second study of gold salts is that of Sigler $e \frac{\mathrm{F}}{\mathrm{F}}$ $a l .{ }^{10}$ In this study 32 patients with early disease were treated with either gold salts or placebo. Five dropped out early (before six weeks), and thees remainder continued for two years. Signs of acute $\vec{\circ}$ inflammation were significantly improved in the gold group, and this group showed a significantlyc. slower rate of progression of erosions. No mentiono is made of comparability of this group with regard to $\overline{0}$ initial ESR.

Two similar studies with penicillamine have beeniv done. The multicentre trial group (five centres) ${ }^{11} \vec{\omega}$ compared 52 patients on $1.5 \mathrm{~g}$ penicillamine per day and 53 patients on placebo. After one year 30 and $38^{\circ}$ patients respectively remained on treatment. Ther- $\overrightarrow{-}$ apeutic failure resulted in nine control withdrawalso but no withdrawals from penicillamine. Disease activity showed greater improvement in the penicillamine treated group. In this study, however, systemic steroids were allowed, and over $60 \% 8 \%$ patients in each group who achieved 12-mont treatment had received such therapy. Williamsäer al. ${ }^{12}$ (11 centres) recently published a placebos

Table 3 Within-group improvement in disease parameters comparing week 0 with weeks 24 and 48 (Wilcoxon matched $\underset{\vec{F}}{\Rightarrow}$ pairs signed rank test [two tailed])

\begin{tabular}{|c|c|c|c|c|c|c|c|c|c|}
\hline & \multicolumn{3}{|l|}{ Study 1} & \multicolumn{2}{|l|}{ Study 2} & \multicolumn{3}{|l|}{ Study 3} & \multirow{2}{*}{ - } \\
\hline & Auranofin & $G S T$ & Placebo & Ketotifen & Placebo & $S A S P$ & $G S T$ & Placebo & \\
\hline \multicolumn{9}{|l|}{24 weeks } & \\
\hline $\mathbf{n}$ & 26 & 23 & 17 & 19 & 11 & 18 & 20 & 19 & ?. \\
\hline ESR & $<0.005$ & NS & NS & NS & NS & $<0.005$ & $<0.005$ & NS & $\dot{\hat{\rho}}$ \\
\hline $\mathrm{Hb}$ & NS & NS & NS & NS & NS & NS & NS & NS & 윽 \\
\hline Platelets & $<0.005$ & $<0.001$ & NS & NS & NS & $<0.001$ & NS & NS & 2 \\
\hline Rheumatoid factor titre & $<0.001$ & $<0.001$ & NS & NS & NS & NS & $<0.05$ & NS & 옥 \\
\hline Articular index & NS & $<0.01$ & NS & $<0.002$ & $<0.003$ & $<0.001$ & $<0.001$ & NS & \\
\hline Pain score & $<0.05$ & $<0.001$ & NS & NS & NS & NS & $<0.005$ & NS & ర \\
\hline Grip strength & NS & NS & NS & NS & NS & $<0.005$ & NS & NS & \\
\hline Early morning stiffness & NS & $<0.05$ & NS & NS & NS & NS & NS & NS & N \\
\hline \multicolumn{9}{|l|}{48 weeks } & n \\
\hline n & 17 & 22 & & & & 12 & 12 & 6 & $n$ \\
\hline ESR & $<0.001$ & $<0.001$ & & & & $<0.001$ & $<0.005$ & NS & \\
\hline $\mathrm{Hb}$ & NS & $<0.05$ & & & & $<0.05$ & $<0.005$ & NS & ס \\
\hline Platelets & & & & & & $<0.005$ & $<0.005$ & NS & 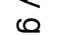 \\
\hline Rheumatoid factor titre & & & & & & NS & NS & NS & 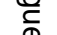 \\
\hline Articular index & $<0.05$ & $<0.01$ & & & & $<0.005$ & $<0.005$ & NS & 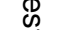 \\
\hline Pain score & $<0.05$ & $<0.001$ & & & & NS & $<0.005$ & NS & 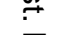 \\
\hline Grip strength & $<0.01$ & $<0.01$ & & & & $<0.005$ & $<0 \cdot 005$ & NS & 7 \\
\hline Early morning stiffness & $<0.005$ & $<0.01$ & & & & $<0.005$ & $<0.005$ & NS & O웅 \\
\hline
\end{tabular}


Table 4 Between-group comparisons of disease parameters (Mann-Whitney $U$ test)

\begin{tabular}{|c|c|c|c|c|c|}
\hline & \multicolumn{2}{|l|}{ Study 1} & \multirow{2}{*}{$\frac{\text { Study } 2}{\text { ketotifen } \mathrm{v} \text { placebo }}$} & \multicolumn{2}{|l|}{ Study 3} \\
\hline & Auranofin v placebo & $G S T \vee$ placebo & & GST $\vee$ placebo & $S A S P \vee$ placebo \\
\hline \multicolumn{6}{|l|}{24 weeks } \\
\hline ESR & NS & $<0.01$ & NS & NS & NS \\
\hline $\mathrm{Hb}$ & NS & NS & NS & NS & NS \\
\hline Platelets & & & NS & NS & NS \\
\hline \multicolumn{6}{|l|}{ Rheumatoid factor titre } \\
\hline Articular index & NS & $<0.05$ & NS & NS & NS \\
\hline Pain score & NS & $<0.001$ & NS & NS & NS \\
\hline Grip strength & NS & NS & NS & NS & NS \\
\hline Early morning stiffness & $<0.05$ & $<0 \cdot 01$ & NS & NS & NS \\
\hline \multicolumn{6}{|l|}{48 weeks } \\
\hline ESR & & & & $<0.05$ & $<0.05$ \\
\hline $\mathrm{Hb}$ & & & & NS & NS \\
\hline Platelets & & & & NS & NS \\
\hline Rheumatoid factor titre & & & & NS & NS \\
\hline Articular index & & & & NS & NS \\
\hline Pain score & & & & $<0.02$ & NS \\
\hline Grip strength & & & & NS & NS \\
\hline Early morning stiffness & & & & NS & NS \\
\hline
\end{tabular}

NS $=$ not significant.

controlled trial of penicillamine, in which 52 patients received placebo, 87 penicillamine $125 \mathrm{mg} /$ day, and 86 penicillamine $500 \mathrm{mg} /$ day. Thirty-weeks' treatment was achieved in 40,70 , and 61 patients respectively, with 5,3 , and 4 patients respectively being withdrawn because of lack of effect. Significantly greater improvement in inflammatory indices was encountered in the high dose penicillamine group as against the placebo group. In this study too, however, systemic corticosteroids were allowed, and $45 \%$ of placebo patients who reached six-months' treatment had received systemic corticosteroids.

In a multicentre (16 centres) comparison of levamisole and placebo, ${ }^{13} 110$ patients received placebo, 124 high dose, and 129 low dose levamisole. Six months of treatment was achieved in 85 , 86 , and 93 patients respectively. Sixteen placebo patients were withdrawn because of lack of effect compared with three high dose and four low dose levamisole treated patients. Levamisole treated patients showed a greater improvement in inflammatory indices than did the controls. Again in this study systemic corticosteroids were allowed, but no mention is made of the number of patients receiving this additional form of therapy.

Two placebo controlled trials of cytotoxic agents in the management of rheumatoid arthritis have been published: one of cyclophosphamide and one of azathioprine. Lidsky et al. ${ }^{4}$ assigned 22 patients to either cyclophosphamide or placebo treatment. Twenty-one patients (10 cyclophosphamide, 11 placebo) completed one year of treatment. Improvement in some clinical indices was found in both groups (not in ESR) and there was no significant difference between the groups. Only one patient received concomitant corticosteroids. The trial of azathioprine $^{15}$ comprised 13 placebo, 15 high dose, and 14 low dose treated patients. Over a six-month period 2,7 , and 4 patients respectively stopped treatment. Only one patient (low dose azathioprine) dropped out because of treatment failure. Patients in all groups showed some significant improvement, but the difference between high dose azathioprine and placebo was significantly different; no patients received corticosteroids.

Information from these studies is summarised in Table 5.

In addition a recent review of a number of studies of the effect of second-line therapy on radiological progression in $\mathrm{RA}^{16}$ indicated that none of these has established a satisfactory answer.

\section{Discussion}

The Concise Oxford Dictionary defines placebo as 'medicine given to humour, rather than cure, the patient'. In clinical trials a placebo group may serve three main purposes: it allows observation of the 'natural history' of the disease; it confounds observer or patient bias; and it indicates any psychological effect which might arise from the taking of medications, frequent clinic visits, etc. It has been argued that in many instances these factors are not relevant 


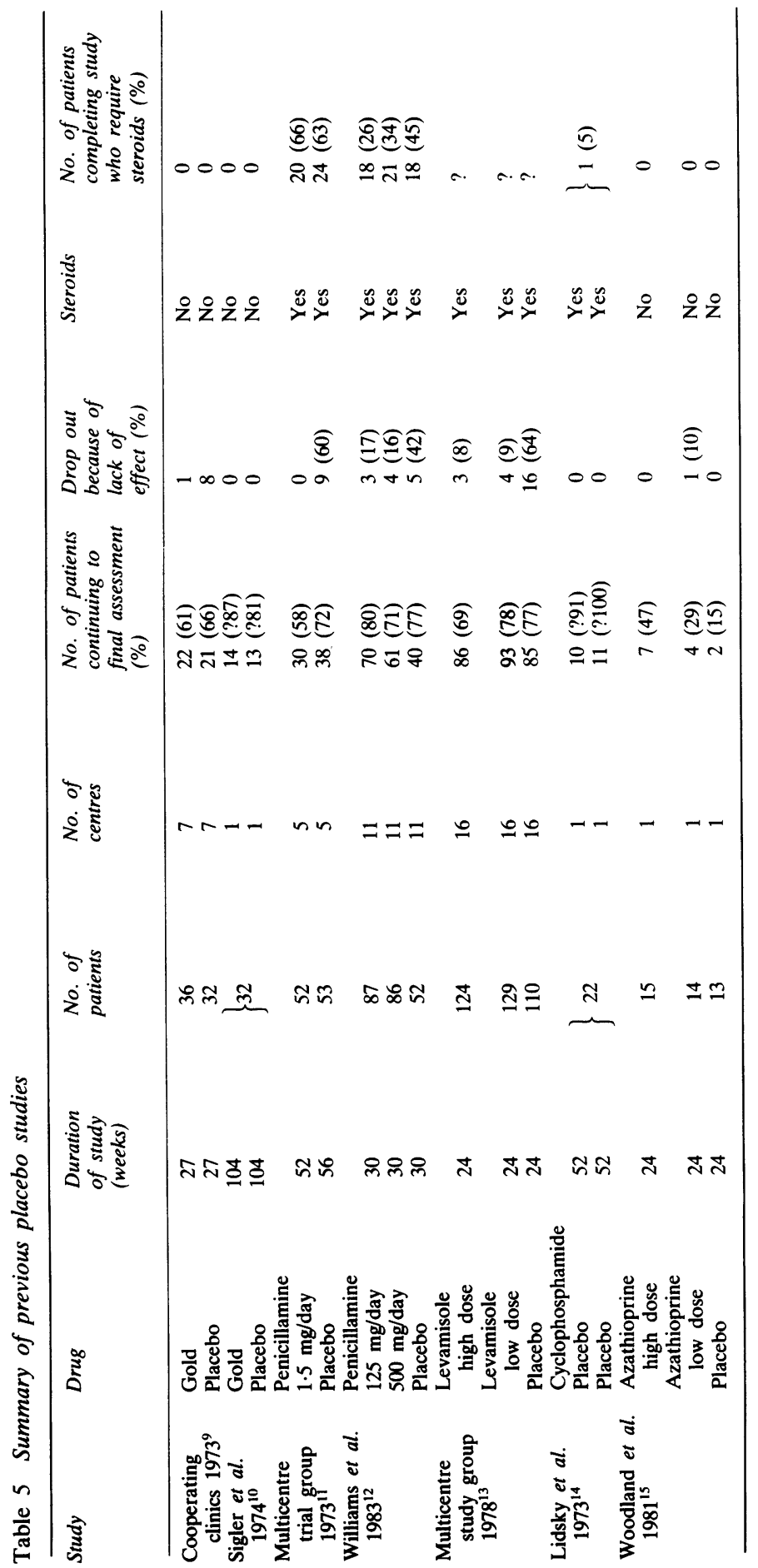


and that a placebo group is unnecessary, unethical, or even illegal. ${ }^{17} 18$ In the field of long-term assessment of second-line agents in rheumatoid arthritis it would seem desirable if not necessary to maintain an adequate placebo group over a long-term period to answer the question: 'Do second-line agents affect the outcome of rheumatoid disease?' Unfortunately the data presented here suggest that the maintenance of an adequate placebo group for one year (which is not long enough to answer the question posed) is not a realistic proposition, and in total only seven $(8 \%)$ out of 90 placebo treated patients attained one year of treatment: even in the study which maintained the largest placebo group only $20 \%$ stayed on for one year. Review of the literature reveals only three studies ${ }^{91014}$ where adequate (though numerically small) placebo groups could be maintained for one year or longer without concomitant administration of corticosteroids (ignoring Lidsky's one patient on steroids whose treatment allocation we do not know). These studies had surprisingly low drop-out rates for both active treatment and placebo groups, and in Sigler's study information about drop outs and pretreatment disease activity is particularly vague. In the absence of fuller information it is uncertain whether the results of these studies are reproducible.

As might be expected the small, self-selected group who remained on placebo at 48 weeks represented a biased sample with milder disease than those who stopped placebo and, in the case of sulphasalazine, than those who remained on active drug. It is, therefore very difficult to comment upon between-group comparisons of inflammatory indices, as two groups of patients with different initial values are being compared. It is equally difficult to interpret within-group changes in the placebo group at 48 weeks, as their indices need to change by less in overall terms to achieve normal levels.

The situation with reference to 'second-line' placebo groups in rheumatoid arthritis is clearly different from the situation in many other disciplines. In rheumatoid arthritis patient's and doctor's expectations are raised because of the well documented and clinically apparent short- or medium-term symptomatic benefit provided by second-line drugs. Clinical benefit with such drugs is expected within six months of initiation of therapy. After six months it is customary to pose the question: 'Is therapy helping you, do you wish to continue?' The negatively responding patient will request a change of therapy, and the doctor is obviously obliged to acquiesce to the patient's wish regardless of whether the drug in question is a placebo or a second-line agent which has failed to exert beneficial change in that individual.
This differs from the situation in hypertension or the secondary prevention of myocardial infarction where the end point is clearly measurable, but neither patient nor doctor expects benefit other than the prevention of vascular catastrophes (an easily measurable end point). If an 'intention to treat analysis' is attempted, a number of factors will contribute to an active group which is largely unfulfilled, and a placebo group which is greatly contaminated. These include the availability of alternative second-line agents, the lag phase before they take effect, the 'hang over' benefit after a drug is stopped, the common practice of having a 'wash out' period between drugs, the high drop-out rate from active agents because of toxicity, and the fact that stopping placebo because of lack of effect results, almost by definition, in the commencement of a second-line drug.

The need for a control group is clear but how might the practical obstacles be overcome? A number of possibilities have been suggested:

(1) Larger numbers - this might give a placebo group sufficiently large to analyse after four to five years, though not even this is certain. However, available results suggest this would not be an unbiased placebo group.

(2) Patients who refuse second-line treatment. In a recent radiological study ${ }^{19}$ only 10 patients who consistently refused second-line therapy could be identified over a two-year period, and most of them subsequently accepted this therapy. This group did not differ from the treatment groups in initial inflammatory indices and failed to show an improvement in these indices over two years. Again, however, this is a biased self-selected group.

(3) Physicians who do not use second-line therapy. Do such rheumatologists exist? If so, do general practitioners who know of their policy refer their worst rheumatoid patients to other physicians? Sequential data are unlikely to be available on untreated patients in the community.

Thus while the need for long-term placebo controlled trials of second-line agents cannot be disputed, experience from this centre and review of the available literature suggest that such studies cannot be done by conventional means. Rheumatologists have been criticised for failing to obtain the relevant data despite treatment of numerous patients. It is not without irony that the degree of medium-term benefit obtained represents possibly the greatest obstacle to long-term assessment. Critics abound: are there any, however, who have a workable solution? 


\section{References}

1 Fraser T N. Gold treatment in rheumatoid arthritis. Ann Rheum Dis 1945; 4: 71-5.

2 The research subcommittee of the Empire Rheumatism Council. Gold therapy in rheumatoid arthritis. Report of a multicentre controlled trial. Ann Rheum Dis 1960; 19: 95-119.

3 Lewis D, Capell H A. Is auranofin preferable to gold sodium aurothiomalate in the management of rheumatoid arthritis? In: Auranofin. Amsterdam: Excerpta Medica, 1983, 147-54.

4 Teh L G, Madhok R, Capell H A. Does the addition of ketotifen to non-steroidal anti-inflammatory drugs confer any additional benefit in rheumatoid arthritis? Br J Clin Pharmacol 1984; 17: 157-9.

5 Pullar T, Hunter J A, Capell H A. Sulphasalazine in rheumatoid arthritis: a double blind comparison of sulphasalazine with placebo and sodium aurothiomalate. $\mathrm{Br}$ Med $J$ 1983; 287: 1102-4.

6 Lewis D, Capell H A. Oral gold: A comparison with placebo and with intramuscular sodium aurothiomalate. Clin Rheumatol in press.

7 Ropes M W, Bennett G A, Cob S, Jacox R, Jessar R A. 1958 revision of diagnostic criteria for rheumatoid arthritis. Arthritis Rheum 1959; 2: 16-20.

8 Mallya R K, Mace B E W. The assessment of disease activity in rheumatoid arthritis using multivariate analysis. Rheumatol Rehabil 1981; 20: 14-7.

9 The Co-operating Clinic Committee of the ARA. A controlled trial of gold salt therapy in rheumatoid arthritis. Arthritis Rheum 1973; 16: 353-8.

10 Sigler J W, Bluhm G B, Duncan H, et al. Gold salts in the treatment of rheumatoid arthritis. Ann Intern Med 1974; 80: 21-6.

11 Multicentre Trial Group. Controlled trial of $\mathrm{D}(-)$ penicillamine in severe rheumatoid arthritis. Lancet 1973 ; i: $275-80$.

12 Williams H J, Ward J R, Reading J C, et al. Low dose $\overline{\bar{S}}$ D-penicillamine therapy in rheumatoid arthritis. Arthritis $\mathbb{D}$ Rheum 1983; 26: 581-92.

13 Multicentre Study Group. Levamisole in rheumatoid arthritis. ڤ్ Lancet 1978; ii: 1007-12.

14 Lidsky M D, Sharp J T, Billings S. Double-blind study of $\vec{\circ}$ cyclophosphamide in rheumatoid arthritis. Arthritis Rheum 1973; 16: 148-53.

15 Woodland J, Chaput de Saintouge D M, Evans S J W, Sharman V L, Currey H L F. Azathioprine in rheumatoid arthritis: double blind study of full versus half dose versus placebo. Ann Rheum Dis 1981; 40: 355-9.

16 Ianuzzi L, Dawson N, Zien N N, Kushner I. Does drug therapy N show radiographic deterioration in rheumatoid arthritis. $N$ Engl $\vec{\omega}$
$J$ Med 1983; 309: 1023-8.

17 Ritter J M. Placebo-controlled, double-blind clinical trials can $\mathrm{O}$ impede medical progress. Lancet 1980; i: 1126-7.

18 Bierkhardt R, Kienle G. Controlled clinical trials and medical $\rightarrow$ ethics. Lancet 1978; ii: 1356-9.

19 Pullar T, Hunter J A, Capell H A. Does second line therapy affect the radiological progression of rheumatoid arthritis? Ann Rheum Dis 1984; 43: 18-23. 